\title{
A Dilemma in the Extreme Low-placed Venus A-Valve in a Cardiogenic Shock Patient
}

\author{
Mi Chen ${ }^{1}$, honglei zhao ${ }^{2}$, Yan Ding ${ }^{1}$, and Lizhong Sun ${ }^{1}$ \\ ${ }^{1}$ Beijing Anzhen Hospital, Capital Medical University \\ ${ }^{2}$ Beijing An Zhen Hospital, Capital Medical University
}

November 4, 2020

\begin{abstract}
A 64-year-old man with severe aortic stenosis and mitral regurgitation presented to our emergency. He had a New York Heart Association class IV symptoms with EuroSCORE II of 20\%. Heart team decide to perform an urgent TAVR. The patient commenced cardiogenic shock in operation room. A Venus $22 \mathrm{~mm}$ balloon and A $26 \mathrm{~mm}$ Venus A-Valve were performed immediately. Aortography and TEE showed a deep implantation, moderate to severe "supra-skirt" paravalvular aortic regurgitation (PAR) and mild prosthetic aortic valve stenosis. After evaluating the hemodynamic tolerability of PAR and the initial mitral regurgitation, the heart team decided to proceed with aortic valve replacement and mitral valve replacement rather than valve-in-valve TAVR.
\end{abstract}

A Dilemma in the Extreme Low-placed Venus A-Valve in a Cardiogenic Shock Patient

Mi Chen, $\mathrm{MD}^{1,2}$, Honglei Zhao MD,PhD ${ }^{1}$, Yan Ding, $\mathrm{MD}^{1}$, Lizhong Sun, MD,PhD ${ }^{1 *}$

${ }^{1}$ Department of Cardiac Surgery, Beijing Anzhen Hospital, Capital Medical University, Beijing, 10029

${ }^{2}$ Department of Cardiac Surgery, University Hospital of Zurich, Zurich

Running title: Extreme Low-placed Venus A-Valve

Correspondence to:

Lizhong Sun MD, PhD

Department of Cardiac Surgery, Beijing Anzhen Hospital, Capital Medical University, Beijing

Anzhen Road 2, Beijing, China

Email: lizhongsun@foxmail.com

Tel: (010) 64456613

Authors' contributions:

Concept, clinical studies and manuscript preparation: Mi Chen; Image collect and analysis: Yan Ding, Manuscript review: Honglei Zhao, Lizhong Sun

\section{Keywords:}

Transcatheter aortic valve replacement, TAVR, paravalvular aortic regurgitation

Data availability statement:

The data used to support the findings of this study are available from the corresponding author upon request. 


\section{Funding Statement:}

This study was supported in part by the National Key Technologies Research and Development Program (No.2015BAI12B03)

\section{Conflict of Interest: None}

\section{Clinical trial registration: None}

We presented a case of TAVR with an extreme low-placed Venus A-Valve in a cardiogenic shock patient with the approval from institutional review board and informed consent. A 64-year-old man with a history of hypertension, gastrointestinal bleeding and chronic kidney disease presented to our emergency complaining of orthopnea and fatigue with progressive aggravation in the last one month. He had acute upper gastrointestinal bleeding and underwent medical treatment one month ago. On physical examination we documented a mid-systolic murmur along the upper right sternal border and pansystolic murmur over the apex. He was in heart failure with coarse crackling and wheezing in both lungs. He had New York Heart Association class IV symptoms with EuroSCORE II of $20 \%$. He had a lean figure with a height of $175 \mathrm{~cm}$ and a weight of 53 $\mathrm{Kg}$.

Transthoracic echocardiography (TEE) demonstrated severe aortic stenosis (AS) and severe mitral regurgitation (MR) with left ventricular ejection fraction of $46 \%$ (left ventricular end-diastolic diameter: $58 \mathrm{~mm}$, left ventricular end-systolic diameter: $45 \mathrm{~mm}$, aortic valve mean pressure gradient: $50 \mathrm{mmHg}$, aortic valve Vmax: $455 \mathrm{~cm} / \mathrm{s}$, mitral valve regurgitation: $3+$ ). Mitral valve was showed with poor coaptation of the leaflets. Contrast-enhanced computed tomography $(\mathrm{CT})$-derived annular area perimeter measurements were $428.9 \mathrm{~mm}^{2}$ and $74.8 \mathrm{~mm}$ (Figure 1), respectively, mandating a $22 \mathrm{~mm}$ Venus balloon to predilate and a $26 \mathrm{~mm}$ Venus A-Valve to implant.

The patient's symptoms, frailty, the burden of comorbidities and technical aspects were evaluated by a multidisciplinary heart team. The heart team decided to proceed with urgent TAVR of a Venus A-Valve (Venus MedTech, Hangzhou, China) and evaluated the mitral regurgitation after TAVR to determinate the staged therapy. Preoperative management included inotropic therapy, morphine injection and blood transfusion. Procedure was performed under general anesthesia in hybrid operating room. Immediately following exposure of right femoral artery, the systolic pressure failed to rise above $40 \mathrm{mmHg}$. Balloon aortic valvuloplasty was performed using a $22 \mathrm{~mm}$ Venus balloon in a short time. After pre-dilation, the patient developed shock and arrested. Cardiopulmonary resuscitation was commenced. Meanwhile the $26 \mathrm{~mm}$ Venus A-Valve was deployed. Extracorporeal circulation was established. Aortography and TEE illustrated an extreme low implantation (Figure 1) and moderate to severe "supra-skirt" paravalvular aortic regurgitation (PAR) (Figure 2 and Figure 3$)^{(1)}$. TEE in short-axis view showed a moderate to severe PAR originated from the right coronary cusp and mild prosthetic aortic valve stenosis (aortic valve mean pressure gradient: $29 \mathrm{mmHg}$, aortic valve Vmax: $339 \mathrm{~cm} / \mathrm{s}$ ). After evaluating the hemodynamic tolerability of PAR, moderate prosthesis-patient mismatch ${ }^{(2)}$, the initial severe mitral regurgitation and potential influence for the movement of the anterior leaflet of mitral valve due to the low deployment, the heart team decided to proceed with aortic valve replacement and mitral valve replacement rather than valve-in-valve TAVR ${ }^{(3)}$. A median sternotomy was done and Venus A-Valve was removed after infiltration by ice water (Figure 4). A 25mm St. Jude Medical mechanical mitral prosthesis and a $19 \mathrm{~mm}$ St. Jude Medical Regent aortic prosthesis were implanted (St Jude Medical, Inc., St Paul, Minn, USA). The cross-clamping time was 105 min and the cardiopulmonary bypass time was $245 \mathrm{~min}$.

TAVR may be an option for patients in high surgical risk with cardiogenic shock (CS) and severe aortic stenosis. The final depth of the Venus A-Valve bioprosthesis is the predictor of paravalvular aortic regurgitation and is associated with prosthesis-patient mismatch (P-PM). P-PM is an essential determinant of morbidity and mortality following TAVR. A widespread and practical percutaneous technique to manage the implant failure of TAVR is required to avoid the surgical bailout. 


\section{References}

1. Pibarot P, Hahn RT, Weissman NJ, Monaghan MJ. Assessment of paravalvular regurgitation following TAVR: a proposal of unifying grading scheme. JACC Cardiovasc Imaging. 2015;8(3):340-60.

2. Jilaihawi H, Chin D, Spyt T, Jeilan M, Vasa-Nicotera M, Bence J, et al. Prosthesis-patient mismatch after transcatheter aortic valve implantation with the Medtronic-Corevalve bioprosthesis. European heart journal. 2010;31(7):857-64.

3. Ussia GP, Barbanti M, Ramondo A, Petronio AS, Ettori F, Santoro G, et al. The valve-in-valve technique for treatment of aortic bioprosthesis malposition an analysis of incidence and 1-year clinical outcomes from the italian CoreValve registry. Journal of the American College of Cardiology. 2011;57(9):1062-8.

\section{Figure 1}

As aortography demonstrated the distal part of the frame is $13 \mathrm{~mm}$ below the nadir of non-coronary cusp (NCC) and $18 \mathrm{~mm}$ below the nadir of left coronary cusp (LCC), whereas the new bioprosthetic aortic annulus is in the height of $15 \mathrm{~mm}$ of the frame (yellow line). The white line indicates the aortic annulus level.

\section{Figure 2}

The long-axis view of TEE showed a moderate to severe paravalvular regurgitation and a potential influence on the movement of the mitral anterior leaflet due to the low-placed flame.

\section{Figure 3}

The short-axis view of TEE showed a "supra-skirt" aortic regurgitation originated from the right coronary cusp.

\section{Figure 4}

The $26 \#$ Venus A-Valve was removed after the infiltration by ice water. 

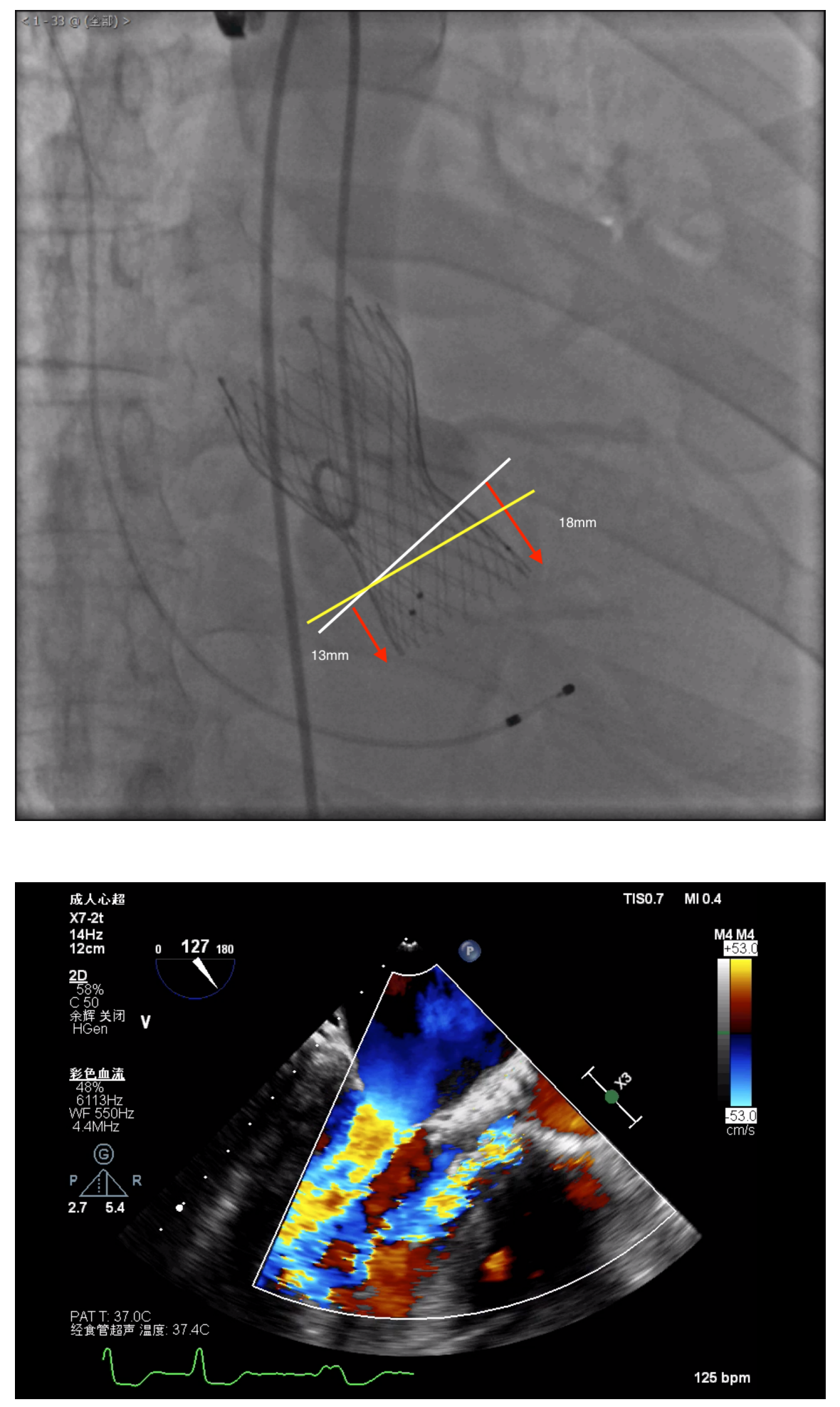


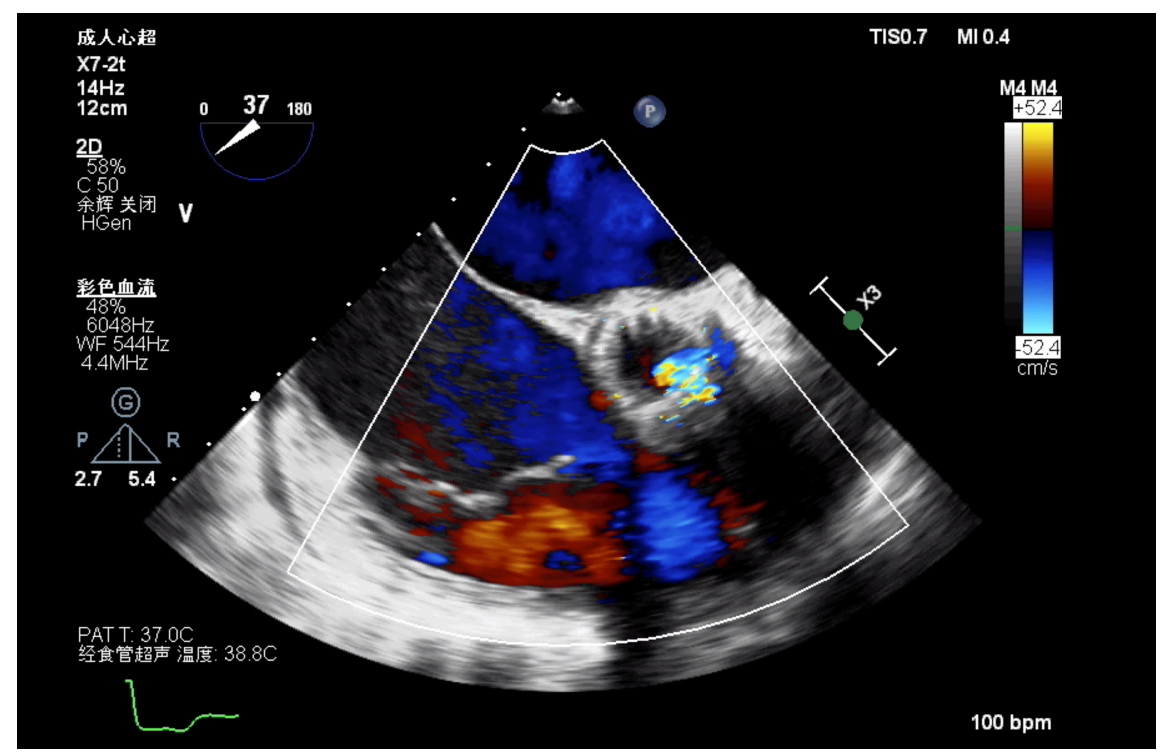




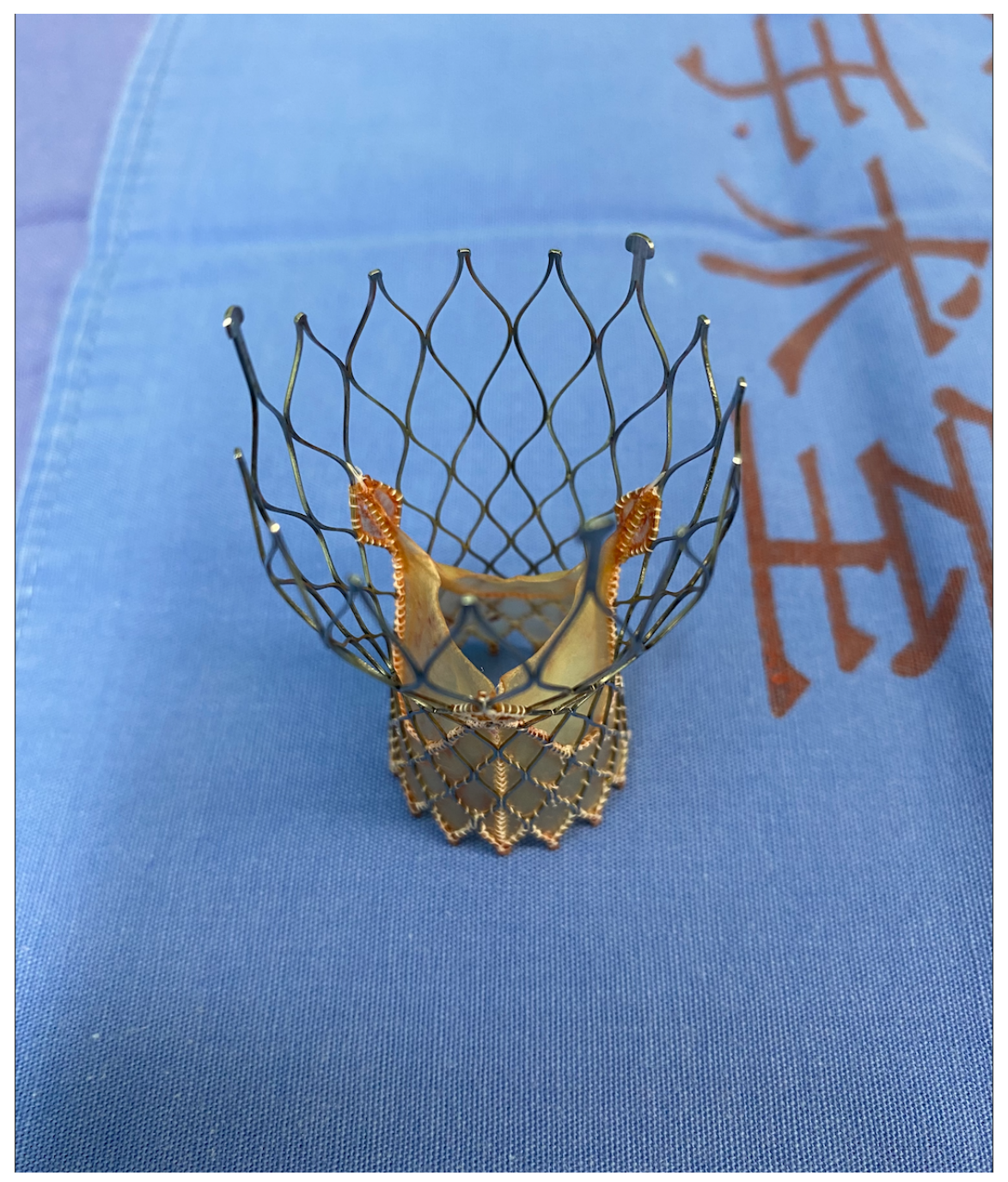

